Metallophysics and Advanced Technologies

металофіз. новітні технол.

Metallofiz. Noveishie Tekhnol.

2019 , vol. 41 , No. 4 , pp. $417-425$

https://doi.org/10.15407/mfint.41.04.0417

Reprints available directly from the publisher
(C) 2019 G. V. Kurdyumov Institute for Metal Physics, National Academy of Sciences of Ukraine Published by license under the G. V. Kurdyumov Institute for Metal PhysicsN.A.S. of Ukraine Publishers imprint. Printed in Ukraine.

\title{
ELECTRONIC STRUCTURE AND PROPERTIE
}

PACSnumbers: 42.70.Hj, 74.25.N-, 74.45.+c, 74.50.+r, 74.81.Fa, 85.25.Am, 85.25.Dq

\section{Фазова синхронізація у системі паралельних нанорозмірних джозефсонівських контактів в зовнішньому високочастотному полі}

\author{
О. Л. Касаткін, А. П. Шаповалов", В. Є. Шатернік, С. І. Футимський \\ Інститут металофізики ім. Г. В. Курдюмова НАН України, \\ бульв. Академіка Вернадського, 36 , \\ 03142 київ, Україна \\ "Iнститут надтвердих матеріалів іл. В. М. Бакуля НАН України, \\ вул.Автозаводська, 2 , \\ 04074 Київ, Україна
}

Показано принципову можливість фазової синхронізації у системі паралельно з'єднаних нанорозмірних джозефсонівських контактів у нестаціонарному режимі за допомогою зовнішнього високочастотного електромагнітного поля. Знайдено умови, що накладаються на амплітуду і частоту зовнішнього поля та забезпечують виникнення синхронізованого режиму генерації електромагнітного випромінювання в такій системі джозефсонівських осциляторів.

Ключові слова: слабкозв'язані надпровідники, макроскопічна квантова когерентність, критичний струм, ефект Джозефсона, джозефсонівська генерація, фазова синхронізація.

Показана принципиальная возможность фазовой синхронизации в системе параллельно соединённых наноразмерных джозефсоновских контактов в нестационарном режиме с помощью внешнего высокочастотного

Corresponding author: Olexandr Leonidovych Kasatkin

E-mail: al_kas@i.ua

G.V. Kurdyumov Institute for Metal Physics, N.A.S. of Ukraine,

36 Academician Vernadsky Blvd., UA-03142 Kyiv, Ukraine

"V. M. Bakul Institute for Superhard Materials, N.A.S. of Ukraine,

2 Avtozavods'ka Str., UA-04074 Kyiv, Ukraine

Citation: O. L. Kasatkin, A. P. Shapovalov, V. E. Shaternik, and S. I. Futimskii, Phase Synchronization in a System of Parallel Connected Nano-Sized Josephson Contacts by External High-Frequency Field, Metallofiz. Noveishie Tekhnol., 41, No. 4: 417-425 (2019), DOI: 10.15407/mfint.41.04.0417. 
электромагнитного поля. Найдены накладываемые на амплитуду и частоту внешнего поля условия, обеспечивающие возникновение синхронизированного режима генерации электромагнитного излучения в такой системе джозефсоновских осцилляторов.

Ключевые слова: слабосвязанные сверхпроводники, макроскопическая квантовая когерентность, критический ток, эффект Джозефсона, джозефсоновская генерация, фазовая синхронизация.

The principal possibility of phase synchronization in a system of parallelconnected nanoscale Josephson contacts in a non-stationary mode due to external high-frequency electromagnetic field is shown. The conditions imposed on the amplitude and frequency of the external field are found for the occurrence of a synchronized electromagnetic field generation in such system of Josephson oscillators.

Key words: weakly coupled superconductors, macroscopic quantum coherence, critical current, Josephson effect, Josephson generation, phase synchronization.

(Отрилано 16 листопада 2018 р.)

\section{1. ВСТУП}

Ефект Джозефсона у слабкозв'язаних надпровідниках є одним 3 найбільш цікавих проявів макроскопічної квантової когерентності, притаманної надпровідному стану. 3 цим ефектом пов'язана велика кількість експериментальних і теоретичних досліджень, які мають надзвичайне значення як для фундаментальної фізики надпровідності, так і для іï прикладних аспектів $[1,2]$. Зокрема, цей ефект лежить в основі роботи унікальних пристроїв надпровідної електроніки, таких як надчутливі приймачі та детектори слабких електромагнітних полів на основі СКВІД-ів $[1,2]$ або елементна база для створення надпровідних суперкомп'ютерів та квантових комп'ютерів (RSFQ логіка, надпровідні кубіти) [3, 4]. Особливий інтерес представляє задача створення на основі ефекту Джозефсона в надпровідних контактах реальних генераторів когерентного випромінювання ТГц-діапазону, частота випромінювання яких може регулюватися постійною напругою на контакті. Ця прикладна задача, цілком очевидно, існує вже багато років, з моменту відкриття ефекту Джозефсона, проте досі залишається невирішеною. Останнім часом намітився деякий прогрес у створенні таких джозефсонівських генераторів з помітним рівнем потужності завдяки використанню в якості джозефсонівських контактів природних джозефсонівських середовищ - квазідвовимірних шаруватих високотемпературних надпровідних сполук, таких як монокристали $\mathrm{Bi}-\mathrm{Sr}-\mathrm{Ca}-\mathrm{Cu}-\mathrm{O}$, які з точки зору електродинаміки представляють собою стопку джозе- 
фсонівських контактів між двовимірними надпровідними шарами $\mathrm{Cu}-\mathrm{O}[5,6]$. Для досягнення прийнятного для практичного застосування рівня потужності високочастотного випромінювання слід застосовувати одночасно багато когерентно випромінюючих джозефсонівських контактів. При цьому виникає задача їхньої синхронізації. Ця задача вирішувалася вже досить давно для ланцюжків послідовно з'єднаних джозефсонівських контактів [7,8], проте й досі залишається актуальною і вирішується в багатьох роботах для різних типів джозефсонівських матриць з послідовним або паралельним з'єднанням окремих джозефсонівських контактів і різними типами зв'язків між ними [9-18].

В даній роботі розглядається можливість фазової синхронізації в системі паралельно з'єднаних джозефсонівських контактів, що виникають у нанорозмірних провідних містках (металевих закоротках) між двома надпровідними плівками, розділеними шаром діелектрика, або в планарних контактах $\mathrm{S}-\mathrm{Sm}(\mathrm{M})-\mathrm{S}-$-типу із проміжним шаром напівпровідника $(\mathrm{Sm})$ із нанорозмірними кластерами нормального металу (M), крізь які може відбуватись тунелювання куперівських пар між надпровідними обкладинками $\mathrm{S}[19,20]$. Реалізацію саме такої конфігурації джозефсонівських контактів у надпровідних гетероструктурах (нанорозмірні провідні містки між двома надпровідними плівками) спостерігали автори [21] при дослідженні MoRe-

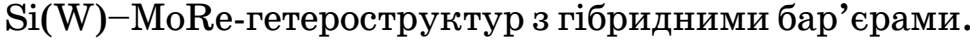

\section{2. ЕФЕКТ ДЖОЗЕФСОНА В СИСТЕМІ ПАРАЛЕЛЬНИХ НАДПРОВІДНИХ МІСТКІВ}

Розглянемо систему паралельних джозефсонівських контактів у вигляді одновимірних нанорозмірних провідних містків у ізолюючому шарі в контактах S-I-S-типу (металевих закороток у діелектричному прошарку), або контактів типу $\mathrm{S}-\mathrm{Sm}(\mathrm{M})-\mathrm{S}$ з напівпровідниковим бар'єром, який містить кластери нормального металу [19, 20] (див. рис. 1). Берегами контактів є суцільні надпровідні плівки, в яких надпровідний стан може бути описаний параметрами порядку $\psi_{1,2}=\sqrt{n_{s} / 2} \exp \left(i \chi_{1,2}(\mathbf{r})\right)$, а густина надплинного струму в берегах: $j_{s, i}(r)=n_{s} e v_{s, i}(\mathbf{r}), i=1,2$, де $v_{s, i}$ - швидкість надплинного конденсату куперівських пар:

$$
v_{s, i}(\mathbf{r})=\frac{1}{2 m}\left(\hbar \nabla \chi_{i}(\mathbf{r})-2 e A(\mathbf{r})\right) .
$$

Струм через джозефсонівський контакт у каналі, розташованому в точці $\mathbf{r}_{k}$ площини плівки, визначається різницею фаз $\varphi\left(\mathbf{r}_{k}\right)=\chi_{1}\left(\mathbf{r}_{k}\right)-\chi_{2}\left(\mathbf{r}_{k}\right)$ в цій точці (див. рис. 1$)$ : 


$$
I_{J}\left(\varphi\left(\mathbf{r}_{k}\right)\right)=I_{c, k} \sin \varphi\left(\tilde{\mathbf{r}}_{k}\right), \tilde{\mathbf{r}}_{k}=\frac{\mathbf{r}_{k}}{r_{k}} \sqrt{r_{k}^{2}+\delta^{2}},
$$

$\delta-$ ефективна ширина каналу. Розподіл струму в кожному з берегів має задовольняти рівнянню неперервності, $\operatorname{div} j_{s, i}(r)-\partial Q_{s, i} / \partial t=0$, де витікання заряду здійснюється через джозефсонівські контакти в містках. Таким чином, у даному випадку рівняння неперервності може бути записано у вигляді:

$$
n_{s} e \frac{\hbar}{2 m} \nabla^{2} \chi_{i}(\mathbf{r})-\sum_{k} I_{J}\left(\varphi\left(\mathbf{r}_{k}\right)\right) \delta\left(\mathbf{r}-\mathbf{r}_{k}\right)=0,(i=1,2),
$$

звідки витікає рівняння для розподілу різниці фаз $\varphi(\mathbf{r})$ в площині берегів:

$$
\nabla^{2} \varphi(\mathbf{r})-\sum_{k} P\left(\mathbf{r}_{k}\right) \delta\left(\mathbf{r}-\mathbf{r}_{k}\right)=0
$$

де

$$
P\left(\mathbf{r}_{k}\right)=\frac{4 m}{n_{s} e \hbar} I_{J}\left(\varphi\left(\mathbf{r}_{k}\right)\right)=\frac{4 m}{n_{s} e \hbar} I_{c, k} \sin \varphi\left(\tilde{\mathbf{r}}_{k}\right) .
$$

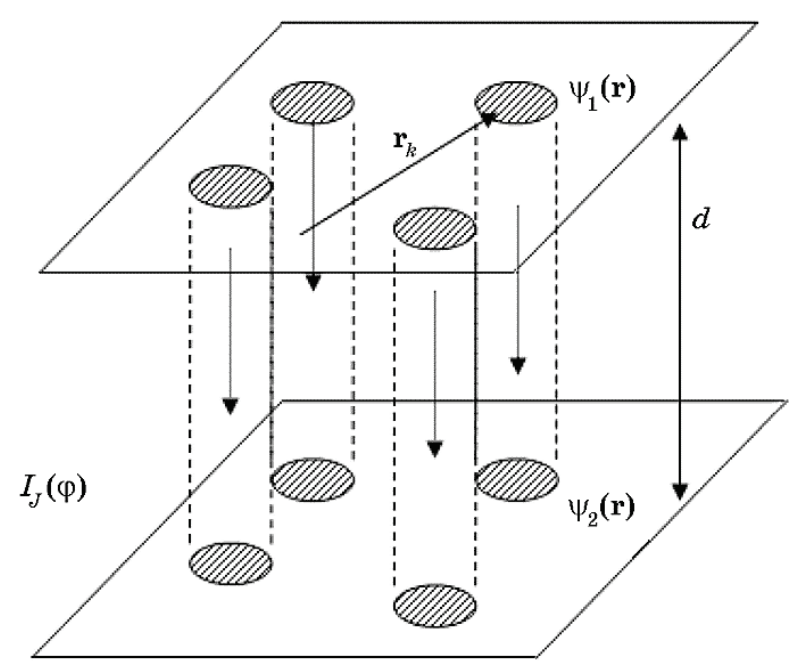

Рис. 1. Схематичне зображення системи паралельно з'єднаних джозефсонівських нанорозмірних містків, що розглядається в роботі.

Fig. 1. Schematic image of the system of parallel-connected nanoscale Josephson bridges which is considered in the present work. 
У двовимірному випадку, який тут розглядається, рівняння (4), (5) мають розв'язок:

$$
\begin{aligned}
& \varphi(\mathbf{r})=\sum_{k} \frac{P\left(\mathbf{r}_{k}\right)}{2 \pi} \ln \left(\left|\mathbf{r}-\mathbf{r}_{k}\right|\right)=\sum_{k} \frac{4 m}{n_{s} e h} I_{J}\left(\varphi\left(\mathbf{r}_{k}\right)\right)= \\
& =\sum_{k} \frac{4 m}{n_{s} e h} I_{c, k} \ln \left(\left|\mathbf{r}-\mathbf{r}_{k}\right|\right) \sin \varphi\left(\tilde{\mathbf{r}}_{k}\right)=\iint_{S} d^{2} \mathbf{r}^{\prime} \ln \left(\left|\mathbf{r}-\mathbf{r}^{\prime}\right|\right) F\left(\mathbf{r}^{\prime}\right) \sin \varphi\left(\tilde{\mathbf{r}}^{\prime}\right), \\
& F(\mathbf{r})=\sum_{k} \frac{4 m}{n_{s} e h} I_{c, k} \delta\left(\mathbf{r}-\mathbf{r}_{k}\right) .
\end{aligned}
$$

Рівняння (6) є самоузгодженим рівнянням для розподілу $\varphi(\mathbf{r})$ в площині берегів. При цьому повний джозефсонівський струм, що протікає крізь систему містків $I_{J}=\sum_{m} I_{c, m} \sin \varphi\left(\tilde{\mathbf{r}}_{m}\right)$, де $\varphi\left(\tilde{\mathbf{r}}_{m}\right)$ визначається розв'язком (6). При цьому критичний струм Джозефсона можна визначити як $I_{J, c}=\left.\max \right|_{\left\{\varphi_{0, k}\right\}} I_{J}\left\{\varphi_{k}\right\}$.

\section{3. СИНХРОНІЗАЦІЯ ДЖОЗЕФСОНІВСЬКИХ КОНТАКТІВ ВИСОКОЧАСТОТНИМ ПОЛЕМ}

Розглянемо нестаціонарний режим такої системи паралельно увімкнених джозефсонівських контактів в присутності високочастотного поля. Для простоти будемо вважати, що на контактах задається напруга: $V(t)=V_{0}+V_{\sim}(t)$, що призводить до нестаціонарного ефекту Джозефсона в містках $[1,2]$ :

$$
\varphi_{i}=\omega_{j}+\Omega_{i}(t), \omega_{j}=\frac{2 e V_{0}}{\hbar}, \Omega_{i}(t)=\frac{2 e V_{i \sim}(t)}{\hbar},
$$

де $\omega_{i}-$ джозефсонівська частота, що визначається постійною напругою $V_{0}$ на контакті, а $\Omega(t)$ - модуляція цієї джозефсонівської частоти, пов'язана з впливом зовнішнього поля $V_{\text {A }}(t)$. Виберемо спеціальну форму цієї модуляції, а саме:

$$
\Omega_{i}(t)=\Omega_{\sim} \sin \left(\omega t-\varphi_{i}(t)\right)=\Omega_{\sim} \sin \theta_{i}(t), \theta_{i}(t)=\omega t-\varphi_{i}(t) \sim\left(\omega-\omega_{j}\right) t .
$$

Такий вид модуляції $\Omega(t)$ виглядає як сигнал гетеродина, і при достатньо близьких частотах $\omega$ та $\omega_{j}$ умову $\left|\left(\omega-\omega_{j}\right) / \omega_{j}\right|<<1$ можна забезпечити схемою підключення джозефсонівського контакту до джерел постійної та змінної напруги. При відсутності індуктивного зв'язку між окремими контактами в системі в рівнянні (8) можна вважати, що $\theta_{i}(t)=\omega t-\varphi_{i}(t)=\left(\omega-\omega_{j}\right) t .3$ рівнянь $(7),(8)$ маємо: 


$$
\begin{aligned}
& \dot{\theta}_{i}(t)=\omega-\dot{\varphi}_{i}(t)=\omega-\omega_{j}-\Omega_{\sim} \sin \theta_{i}(t), \\
& \dot{\theta}_{i}(t)+\Omega_{\sim} \sin \theta_{i}(t)=\omega-\omega_{j} \equiv \Delta \omega .
\end{aligned}
$$

Переходячи в другому 3 рівнянь (9) до безрозмірного часу, $t \rightarrow \tau=\Omega_{\sim} t$, отримуємо:

$$
\dot{\theta}_{i}(\tau)+\sin \theta_{i}(\tau)=\frac{\Delta \omega}{\Omega_{\sim}} \equiv k .
$$

При значеннях параметру $|k|<1$ рівняння (10) має стаціонарне рішення:

$$
\theta_{i}=\arcsin k=\operatorname{const}(t) .
$$

Це рішення означає захват частоти зовнішнім джерелом змінної напруги: $\omega_{j} \rightarrow \omega$ і $\varphi_{i}(t)=\omega t+\varphi_{i 0}$, де $\varphi_{i 0}=-\arcsin k-$ однакове значення для всіх контактів в системі. Тобто, при значеннях параметру $|k|<1$ в рамках цієї спрощеної моделі має досягатись фазова синхронізація i, відповідно, когерентне випромінювання всіх паралельно з'єднаних джозефсонівських контактів [22, 23]. Така синхронізація буде призводити до максимуму амплітуди джозефсонівського струму (6).

При значеннях параметру $|k|>1$ стаціонарні рішення рівняння (10) відсутні. Проте це рівняння можна інтегрувати і отримати залежність $\theta(\tau)$ у вигляді неявної функції:

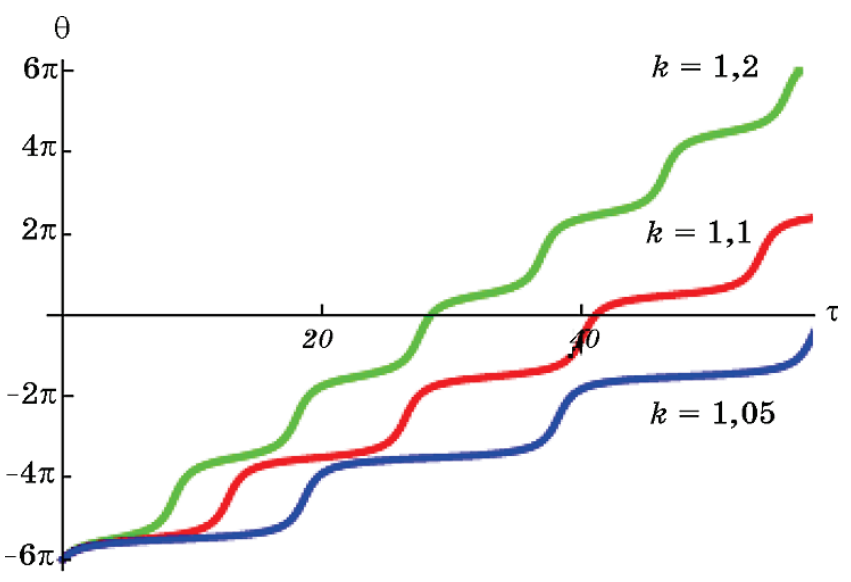

Рис. 2. Залежність $\theta(\tau)$ при трьох різних значеннях $|k|>1$.

Fig. 2. Dependence $\theta(\tau)$ according to Eq. (12) for three different values of parameter $k(|k|>1)$. 


$$
\tau=\int \frac{d \theta}{\dot{\theta}}=\int_{\theta_{m}}^{\theta} \frac{d \theta}{k-\sin \theta}=\left.\frac{2}{\sqrt{k^{2}-1}} \operatorname{arctg}\left[\frac{k \operatorname{tg} \frac{\theta}{2}-1}{\sqrt{k^{2}-1}}\right]\right|_{\theta_{m}} ^{\theta}, \theta_{m}=\theta(\tau=0)
$$

Залежність $\theta(\tau)$ при трьох різних значеннях $|k|>1$ показана на рис. 2. Ця залежність має періодичні стрибки фази, що відповідає явищу биття фазової залежності $\varphi(\tau)=\omega \tau-\theta(\tau)$ із частотою биття $\Omega_{b}=\sqrt{k^{2}-1}[23]$.

\section{4. ВИСНОВКИ}

Досліджено спрощену модель нестаціонарного стану системи паралельно з'єднаних нанорозмірних джозефсонівських контактів у вигляді одновимірних нанорозмірних провідних містків в ізолюючому шарі контактів S-I-S-типу (металевих закоротках у діелектричному прошарку), або контактів типу $\mathrm{S}-\mathrm{Sm}(\mathrm{M})-\mathrm{S}$ з напівпровідниковим бар'єром, який містить кластери нормального металу, крізь які може відбуватися тунелювання куперівських пар електронів. Вважається, що ця система знаходиться у нестаціонарному режимі завдяки прикладеній постійній напрузі $V_{0}$, а також під впливом зовнішнього високочастотного електромагнітного поля. Для певного виду високочастотного збудження знайдено умови, що накладаються на амплітуду і частоту зовнішнього поля та забезпечують виникнення синхронізованого режиму генерації електромагнітного випромінювання в такій системі джозефсонівських осциляторів.

Публікація містить результати досліджень, проведених при грантовій підтримці Держаного фонду фундаментальних досліджень за конкурсним проектом $\Phi 76 / 36725$.

\section{ЦИТОВАНА ЛІТЕРАТУРА}

1. А. Бароне, Дж. Патерно, Эффект Джозефсона (Москва: Мир: 1984).

2. К. К. Лихарев, Введение в динамику джозефсоновских переходов (Москва: Наука: 1985).

3. K. K. Likharev and V. K. Semenov, IEEE Trans. Appl. Supercond., 1: 3 (1991).

4. А. Н. Омельянчук, Е. В. Ильичев, С. Н. Шевченко, Квантовые когерентные явления в джозефсоновских кубитах (Київ: Наукова думка: 2013).

5. T. M. Benseman, K. E. Gray, A. E. Koshelev, W. K. Kwok, U. Welp, H. Minami, K. Kadowaki, and T. Yamamoto, Appl. Phys. Lett., 103: 022602 (2013).

6. E. A. Borodianskyi and V. M. Krasnov, Nat. Commun., 8: 1742 (2017).

7. А. Л. Гудков, М. Ю. Куприянов, А. Н. Самусь, ЖЭТФ, 141: 939 (2012). 
8. V. E. Shaternik, A. P. Shapovalov, and O. Yu. Suvorov, Low Temp. Phys., 43: 877 (2017).

9. A. K. Jain, K. K. Likharev, J. E. Lukens, and J. E. Sauvageau, Phys. Reports, 109: 309 (1984).

10. P. Hadley, M. R. Beasly, and K. Wiesenfeld, Appl. Phys. Lett., 52: 1619 (1988).

11. L. L. Sohn, M. S. Rzchowski, J. U. Free, and M. Tinkham, Phys. Rev. B, 47: 967 (1993).

12. L. L. Sohn, M. T. Tuominen, M. S. Rzchowski, J. U. Free, and M. Tinkham, Phys. Rev. B, 47: 975 (1993).

13. J. K. Harbaugh and D. Stroud, Phys. Rev. B, 56: 8335 (1997).

14. G. Filatrella, N. F. Pedersen, and K. Wiesenfeld, Phys. Rev. E, 61: 2513 (2000).

15. B. R. Trees, V. Saranathan, and D. Stroud, Phys. Rev. E, 71: 016215 (2005).

16. D. Chevriaux, R. Khomeriki, and J. Leon, Phys. Rev. B, 73: 214516 (2006).

17. R. N. Chitra, and V. C. Kuriakose, CHAOS, 18: 023129 (2008).

18. B. C. Daniels, S. T. M. Dissanayake, and B. R. Trees, Phys. Rev. E, 67: 026216 (2003).

19. J. Hindes and C. R. Myers, CHAOS, 25: 073119 (2015).

20. M. A. Galin, E. A. Borodyanskii, V. V. Kurin, L. A. Shershevski, N. K. Vdovicheva, V. M. Krasnov, and A. M. Klushin, Phys. Rev. Applied, 9: 054032 (2018).

21. V. E. Shaternik, A. P. Shapovalov, T. A. Prikhna, O. Y. Suvorov, M. A. Skorik, V. I. Bondarchuk, and V. E. Moshchil, IEEE Trans. Appl.Supercond., 27, No. 4: 1800507 (2017).

22. Henry D. I. Abarbanel, M. I. Rabinovich, and M. M. Sushchik, Introduction to Nonlinear Dynamics for Physicists (World Scientific Publishing Co. Pte. Ltd.:1993).

23. G. V. Osipov and M. M. Sushchik, Phys. Rev. E, 58: 7198 (1998).

\section{REFERENCES}

1. A. Barone and G. Paterno, Physics and Applications of the Josephson Effect (John Willey \& Sons, Inc.: 1982).

2. K. K. Likharev, Vvedenie v Dinamiku Dzhozephsonovskikh Perekhodov [Introduction to the Josephson Junctions Dynamics] (Moscow: Nauka: 1985) (in Russian).

3. K. K. Likharev and V. K. Semenov, IEEE Trans. Appl. Supercond., 1: 3 (1991).

4. A. N. Omel'yanchouk, E. V. Il'yichev, and S. N. Shevchenko, Kvantovye Kogerentnye Yavleniya v Dzhosefsonovskikh Kubitakh [Quantum Coherence Phenomena in Josephson Qubits (Kiev: Naukova Dumka: 2013).

5. T. M. Benseman, K. E. Gray, A. E. Koshelev, W. K. Kwok, U. Welp, H. Minami, K. Kadowaki, and T. Yamamoto, Appl. Phys. Lett., 103: 022602 (2013).

6. E. A. Borodianskyi and V. M. Krasnov, Nat. Commun., 8, Article number 1742 (2017).

7. A. L. Gudkov, M. Y. Kupriyanov, and A. N. Samus', J. Exp. Theor. Phys., 114: 818 (2012).

8. V. E. Shaternik, A. P. Shapovalov, and O. Yu. Suvorov, Low Temp. Phys., 43: 877 (2017). 
9. A. K. Jain, K. K. Likharev, J. E. Lukens, and J. E. Sauvageau, Phys. Reports, 109: 309 (1984).

10. P. Hadley, M. R. Beasly, and K. Wiesenfeld, Appl. Phys. Lett., 52: 1619 (1988).

11. L. L. Sohn, M. S. Rzchowski, J. U. Free, and M. Tinkham, Phys. Rev. B, 47: 967 (1993).

12. L. L. Sohn, M. T. Tuominen, M. S. Rzchowski, J. U. Free, and M. Tinkham, Phys. Rev. B, 47: 975 (1993).

13. J. K. Harbaugh and D. Stroud, Phys. Rev. B, 56: 8335 (1997).

14. G. Filatrella, N. F. Pedersen, and K. Wiesenfeld, Phys. Rev. E, 61: 2513 (2000).

15. B. R. Trees, V. Saranathan, and D. Stroud, Phys. Rev. E, 71: 016215 (2005).

16. D. Chevriaux, R. Khomeriki, and J. Leon, Phys. Rev. B, 73: 214516 (2006).

17. R. N. Chitra, and V. C. Kuriakose, CHAOS, 18: 023129 (2008).

18. B. C. Daniels, S. T. M. Dissanayake, and B. R. Trees, Phys. Rev. E, 67: 026216 (2003).

19. J. Hindes and C. R. Myers, CHAOS, 25: 073119 (2015).

20. M. A. Galin, E. A. Borodyanskii, V. V. Kurin, L. A. Shershevski, N. K. Vdovicheva, V. M. Krasnov, and A. M. Klushin, Phys. Rev. Applied, 9: 054032 (2018).

21. V. E. Shaternik, A. P. Shapovalov, T. A. Prikhna, O. Y. Suvorov, M. A. Skorik, V. I. Bondarchuk, and V. E. Moshchil, IEEE Trans. Appl.Supercond., 27, No. 4: 1800507 (2017).

22. Henry D. I. Abarbanel, M. I. Rabinovich, and M. M. Sushchik, Introduction to Nonlinear Dynamics for Physicists (World Scientific Publishing Co. Pte. Ltd.:1993).

23. G. V. Osipov and M. M. Sushchik, Phys. Rev. E, 58: 7198 (1998). 